\title{
Basic interactions in black hole cosmology
}

\author{
U. V. S. Seshavatharam ${ }^{1}$, S. Lakshminarayana ${ }^{2}$ \\ ${ }^{1}$ Honorary faculty, I-SERVE, Alakapuri, Hyderabad-35, AP, India \\ ${ }^{2}$ Dept. of Nuclear Physics, Andhra University, Visakhapatnam-03, AP, India
}

\section{Email address:}

seshavatharam.uvs@gmail.com (U. V. S. Seshavatharam), lnsrirama@yahoo.com (S. Lakshminarayana)

\section{To cite this article:}

U. V. S. Seshavatharam, S. Lakshminarayana. Basic Interactions in Black Hole Cosmology. American Journal of Astronomy and Astrophysics. Vol. 2, No. 1, 2013, pp. 6-17. doi: 10.11648/j.ajaa.20140201.12

\begin{abstract}
By highlighting the 12 major shortcomings of modern big bang cosmology and reinterpreting the cosmic redshift as a galactic atomic emission phenomenon, the authors made an attempt to develop a possible model of Black hole cosmology in a constructive way. Its validity can be well confirmed from a combined study of cosmological and microscopic physical phenomena. It can be suggested that, there exists one variable physical quantity in the presently believed atomic and nuclear physical constants and "rate of change" in its magnitude can be considered as a "standard measure" of the present "cosmic rate of expansion". The characteristic nuclear charge radius, inverse of the Fine structure ratio, the characteristic reduced Planck's constant seem to increase with cosmic time and there will be no change in the magnitude of Planck's constant. At any cosmic time, 'Hubble length' can be considered as the gravitational or electromagnetic interaction range. With this idea, independent of the reduced Planck's constant, inverse of the Fine structure ratio can be fitted in a cosmological approach. At any cosmic time, the product of 'critical density' and 'Hubble volume' gives a characteristic cosmic mass and it can be called as the 'Hubble mass'. Schwarzschild radius of the 'Hubble mass' again matches with the 'Hubble length'. Most of the cosmologists believe that this is merely a coincidence. Here the authors emphasize the fact that this coincidence is having deep connection with cosmic geometry and the cosmological and microscopic physical phenomena. In this model, forever rotating at light speed, high temperature and high angular velocity small sized primordial cosmic black hole of mass $M_{C} \cong \sqrt{e^{2} / 4 \pi \varepsilon_{0} G}$ gradually transforms into a low temperature and low angular velocity large sized massive primordial cosmic black hole.
\end{abstract}

Keywords: Big Bang Model, Black Holes, Mach’s Principle, Fundamental Interactions, Final Unification

\section{Introduction}

In this paper by highlighting the following 12 major short comings of modern big bang cosmology [1] the authors made an attempt to develop a possible model of Black hole cosmology in a constructive way. It's validity can be well confirmed from a combined study of cosmological and microscopic physical phenomena. One can find detailed information in our published review article [2]. From now onwards instead of focusing on 'big bang cosmology' it is better to concentrate on 'black hole cosmology' [3-5]. According to Tinaxi Zhang [3], the universe originated from a hot star-like black hole with several solar masses and gradually grew up through a supermassive black hole with billion solar masses to the present state with hundred billion-trillion solar masses by accreting ambient materials and merging with other black holes. According to N. J. Poplawski [4], the Universe is the interior of an Einstein-Rosen black hole and began with the formation of the black hole from a supernova explosion in the center of a galaxy. Most recently cosmologists Razieh Pourhasan, Niayesh Afshordi and Robert B. Manna have proposed [5] that the Universe formed from the debris ejected when a four-dimensional star collapsed into a black hole - a scenario that would help to explain why the cosmos seems to be so uniform in all directions.

\section{Major Shortcomings of Modern Big Bang Cosmology}

A) The standard Big Bang model tells us that the Universe exploded out of an infinitely dense point, or singularity. But nobody knows what would have triggered this outburst: the known laws of physics cannot tell us what happened at that moment.

B) Really if there was a 'big bang' in the past, with reference to formation of the big bang as predicted by 
GTR and with reference to the cosmic expansion that takes place simultaneously in all directions at a uniform rate at that time about the point of big bang 'point' of big bang can be considered as the centre or characteristic reference point of cosmic expansion in all directions. In this case, saying that there is no preferred direction in the expanding universe - may not be correct.

C) There is no scientific evidence for the Friedmann's second assumption. We believe it only on the grounds of modesty [6].

D) Dimensionally it is perfectly possible to show that, the dimensions of Hubble's constant and angular velocity are same. If so considering Hubble's constant merely as an expansion parameter may not be correct. Please see the section-5.

E) Even though it was having strong footing, Mach's principle [7] was not implemented successfully. At any given cosmic time, the product of 'critical density' and 'Hubble volume' gives a characteristic cosmic mass and it can be called as the 'Hubble mass'. Interesting thing is that, Schwarzschild radius of the 'Hubble mass' again matches with the 'Hubble length'. Most of the cosmologists believe that this is merely a coincidence. Here the authors emphasize the fact that this coincidence is having deep connection with cosmic geometry and the cosmological physical phenomena.

F) It may be noted that, increased redshifts and increased distances forced Edwin Hubble to propose the Hubble's law $[8,9]$. In fact there is no chance or scope or place for 'galaxy receding'. It is only our belief in its 'given' (Doppler shift based) interpretation. Even then, merely by estimating galaxy distance and without measuring galaxy receding speed, one cannot verify its acceleration. Clearly speaking: two mistakes are happening here. 1) Assumed galaxy receding speed is not being measured and not being confirmed. 2) Without measuring and confirming the galaxy receding speed, how can one say and confirm that it (galaxy) is accelerating. It is really speculative and unfortunate also.

G) During cosmic expansion, assuming past and present galaxies (which actually found to have gigantic structures) as 'points' and guessing photons coming from that galactic point particles seem to be ad-hoc. If light is coming from the atoms of the gigantic galaxy, then redshift can be interpreted as an index of the galactic atomic 'light emission mechanism'. In no way it seems to be connected with 'galaxy receding'.

$\mathrm{H}$ ) If cosmic expansion is continuous and accelerating and redshift is a measure of cosmic expansion, then 'rate of increase in redshift' can be considered as a measure of cosmic 'rate of expansion'. Then there is no possibility to observe a 'constant' red shift. More over the current definition of red shift seems to be ad-hoc and not absolute. Please see section 6. Hence one may not be able to understand or confirm the actual cosmic rate of expansion.

I) According to the modern cosmological approach, bound systems like 'atoms' which found to be the major constituents of galactic matter - will not expand with cosmic expansion/acceleration. As per the present observational data this may be true. It might be the result of ending stage of expansion also. In this regard, without considering and without analysing the past data, one can not come to a conclusion. If it is not possible to collect the past data, theoretically it may be possible to proceed further in this new direction.

J) Even though the whole physics strictly follows the 'constancy of speed of light', cosmic acceleration seems to violate it. This is really doubtful.

$\mathrm{K})$ Drop in 'cosmic temperature' can be considered as a measure of cosmic expansion and 'rate of decrease in cosmic temperature' can be considered as a measure of cosmic 'rate of expansion'. But if rate of decrease in temperature is very small and is beyond the scope of current experimental verification, then the two possible states are: a) cosmic temperature is decreasing at a very slow rate and universe is expanding at a very slow rate and b) there is no 'observable' thermal expansion and there is no 'observable' cosmic expansion.

L) If 'Dark energy' is the major outcome of the 'accelerating universe', it is very important to note that - in understanding the basic concepts of unification or other fundamental areas of physics, role of dark energy is very insignificant. So far no ground based experiment confirmed the existence of dark energy. There is no single clue or definition or evidence to any of the natural physical properties of (the assumed) dark energy.

Based on these short comings the concepts of 'big bang cosmology' can be relinquished. The subject of cosmology is open. If one is willing to think in this new direction, certainly other hidden things can also be surfaced out. Most of the modern cosmologists are enforced with 85 years old Hubble's interpretation. This is the time to re-interpret the Hubble's law and to revise the basics of modern cosmology. Compared to the Big bang model, advantage of Black hole cosmology lies in confirming its validity through atomic and nuclear experimental results.

\section{Black Holes and Black Hole Cosmology}

In the standard cosmology, 'Hubble volume' or 'Hubble sphere' is a spherical region of the Universe surrounding an observer beyond which objects recede from that observer at a rate greater than the speed of light due to the expansion of the Universe. The commoving radius of a Hubble sphere (known as the Hubble radius or the Hubble length) is $\left(c / H_{0}\right)$, where $(c)$ is the speed of light and $\left(H_{0}\right)$ is the Hubble constant. More generally, the term 'Hubble 
volume' can be applied to any region of space with a volume of the order of $(4 \pi / 3)\left(c / H_{0}\right)^{3}$. 'Hubble volume' can be considered as a key tool in cosmology and unification. Please note that at any given cosmic time, the product of 'cosmic critical density' and 'Hubble volume' gives a characteristic cosmic mass and it can be called as the 'Hubble mass'. Interesting thing is that, Schwarzschild radius of the 'Hubble mass' again matches with the 'Hubble length'. Most of the cosmologists believe that this is merely a coincidence. Here the authors emphasize the fact that this coincidence is having deep connection with the cosmic geometry and the cosmological \& microscopic physical phenomena. At any given cosmic time, 'Hubble length' can be considered as the gravitational or electromagnetic interaction range. If one is willing to think in this direction, by increasing the number of applications of Hubble mass and Hubble volume in other areas of fundamental physics like quantum physics, nuclear physics, atomic physics and particle physics slowly and gradually - in a progressive way, concepts of 'Black hole Cosmology' can be strengthened and can also be confirmed [10-27]. If one is able to show the applications of 'Hubble mass' in different areas of fundamental physics, certainly it can be given more significance and top priority compared to the mysterious 'dark energy'.

With reference to the well believed big bang, in the universe there is no centre, there is no preferred direction and there is no rotation. With reference to galactic spinning black holes, it is well confirmed that, there is a center, there is rotation and there is a preferred direction. Considering a $4 \mathrm{D} / 3 \mathrm{D}$ or $3 \mathrm{D}$ star like black hole (that is assumed to be responsible for the cosmic evolution) with no centre, with no preferred direction and with no rotation is not correct. Hence the possible 'new solution' seems to be - to give up the old unanswerable concepts of big bang and to become accustomed with the newly accepted concepts of 4D/3D or $3 \mathrm{D}$ cosmic primordial black hole with center and rotation and see the consequences!

\section{The Proposed Picture of Black Hole Cosmology}

Instead of considering 'star - black hole explosions' and 'higher dimensions', the authors of this paper focused their attention only on the old and famous Mach's principle, 'Hubble volume' and 'primordial evolving black holes'. Some cosmologists use the term 'Hubble volume' to refer to the volume of the observable universe. There is no perfect theory that defines the lower and upper limits of a massive black hole. Most of the theoretical models assume a lower mass limit close to the 'Planck mass'. Astronomers believe that black holes that are as large as a billion solar masses can be found at the centre of most of the galaxies. Here the fundamental questions to be answered are: If the galactic central black hole mass is 10 billion solar masses and density is less than $1 \mathrm{~kg} / \mathrm{m}^{3}$ - with such a small density and large mass, without collapsing - how it is able to hold a gigantic galaxy? What force makes the black hole stable? Recent observations confirm that, instead of collapsing, galactic central black holes are growing faster and spinning with light speed. Even though mass is too high and density is too low, light speed rotation certainly helps in maintaining black hole's stability from collapsing with maximum possible outward radial force of the magnitude close to $\left(c^{4} / G\right)$. If 'black hole geometry' is intrinsic compared to the black hole 'mass' and 'density' parameters, if universe constitutes so many galaxies and if each galaxy constitutes a central growing and fast spinning black hole then considering universe as an 'evolving and light speed rotating primordial black hole' may not be far away from reality.

Based on these points the authors propose the following picture of Black hole cosmology. Forever rotating at light speed, high temperature and high angular velocity small sized primordial cosmic black hole of mass $M_{C} \cong \sqrt{e^{2} / 4 \pi \varepsilon_{0} G}$ gradually transforms into a low temperature and low angular velocity large sized massive primordial cosmic black hole. At any given cosmic time, for the primordial growing black hole universe, its 'Schwarzschild radius' can be considered as its characteristic possible minimum radius and 'constant light speed rotation' will give the maximum possible stability from collapsing. Here $M_{C} \cong \sqrt{e^{2} / 4 \pi \varepsilon_{0} G}$ can be called as the mass of the primordial baby black hole universe. Here 3 important points can be stated as follows.

1. In theoretical physics, particularly in discussions of gravitation theories, Mach's principle is the name given by Einstein to an interesting hypothesis often credited to the physicist and philosopher Ernst Mach. The idea is that the local motion of a rotating reference frame is determined by the large scale distribution of matter. With reference to the Mach's principle and the Hubble volume, at any cosmic time, if 'Hubble mass' is the product of cosmic 'critical density' and the 'Hubble volume', then it can be suggested that, 1) Each and every point in the free space is influenced by the Hubble mass, 2) Hubble volume and Hubble mass play a vital role in understanding the properties of electromagnetic and nuclear interactions and 3) Hubble volume and Hubble mass play a key role in understanding the geometry of the universe. Unless the applications of Hubble volume and Hubble mass in microscopic physics are surfaced out its very hard to strengthen the concepts of black hole cosmology and it is also not possible to abandon the big bang model. It is the reason for interrelating the (currently believed) unrelated microscopic quantities and the cosmic physical parameters. Independent of cosmological observations this procedure helps in developing a unified model of physics and also helps in confirming the proposed 'black hole universe' and 'cosmic rotation' concepts from ground based laboratory experimental results.

2. Starting from an electron to any gigantic galaxy, 
rotation is a common phenomena in atomic experiments and astronomical observations. From Newton's laws of motion and based on the Mach's principle, sitting inside a closed universe, one cannot comment whether the universe is rotating or not. We have to search for alternative means for confirming the cosmic rotation. Recent findings from the University of Michigan [28] suggest that the shape of the Big Bang might be more complicated than previously thought, and that the early universe spun on an axis. A lefthanded and right-handed imprint on the sky as reportedly revealed by galaxy rotation would imply the universe was rotating from the very beginning and retained an overwhelmingly strong angular momentum. An anonymous referee who reviewed the paper for Physics Letters said, "In the paper the author claims that there is a preferred handedness of spiral galaxies indicating a preferred direction in the universe. Such a claim, if proven true, would have a profound impact on cosmology and would very likely result in a "Nobel prize". The consequences of a spinning universe [28-42] seem to be profound and natural. Not only that, with 'constant rotation speed' 'cosmic collapse' can be prevented and can be considered as an alternative to the famous 'repulsive gravity' concept. If so, at any time to have maximum possible stability from collapsing 'constant light speed rotation' can be considered as a constructive and workable concept.

3. Recent observations confirm black hole's light speed rotation. In 2013 February, using NASA's newly launched NuStar telescope and the European Space Agency's workhorse XMM-Newton, an international team observed high-energy X-rays released by a super massive black hole in the middle of a nearby galaxy. They calculated its spin at close to the speed of light: 670 million mph [43].Please note that, for any black hole even though it's mass is too high and density is too low, light speed rotation certainly helps in maintaining its stability from collapsing with maximum possible outward radial force [44] of magnitude $\left(c^{4} / G\right)$. At the beginning of comic evolution if rotation speed was zero and there was no big bang - definitely it will cast a doubt on the stability, existence and angular velocity of the assumed initial primordial cosmic baby black hole. Hence at the beginning also, to guess or define the angular velocity and to have maximum possible stability it is better to assume light speed rotation for the cosmic baby black hole. At present if rate of cosmic expansion is very slow, then rate of decrease in angular velocity will be very small and practically can be considered as zero. Along with (practically) constant angular velocity, at present if constant light speed rotation is assumed to be maintained then cosmic stability will be maximum and rate of change in cosmic size will be practically zero and hence this idea helps us to believe in present Hubble length along with the observed ordered galactic structures and uniform thermal energy density.

\section{The Cosmic 'Critical Density' and its Dimensional Analysis and the Cosmic Rotation}

With a simple derivation it is possible to show that, Hubble's constant $H_{t}$ represents cosmological angular velocity. Authors presented this derivation in their published papers. Assume that, a planet of mass $M$ and radius $\mathrm{R}$ rotates with angular velocity $\omega_{e}$ and linear velocity $v_{e}$ in such a way that, free or loosely bound particle of mass $\mathrm{m}$ lying on its equator gains a kinetic energy equal to potential energy as,

$$
\begin{gathered}
\frac{1}{2} m v_{e}^{2}=\frac{G M m}{R} \\
R \omega_{e}=v_{e}=\sqrt{\frac{2 G M}{R}} \text { and } \omega_{e}=\frac{v_{e}}{R}=\sqrt{\frac{2 G M}{R^{3}}}
\end{gathered}
$$

i.e Linear velocity of planet's rotation is equal to free particle's escape velocity. Without any external power or energy, test particle gains escape velocity by virtue of planet's rotation. Using this idea, 'Black hole radiation' and 'origin of cosmic rays' can be understood. Note that if Earth completes one rotation in one hour then free particles lying on the equator will get escape velocity. Now writing $M=\frac{4 \pi}{3} R^{3} \rho_{e}$

$$
\begin{gathered}
\omega_{e}=\frac{v_{e}}{R}=\sqrt{\frac{8 \pi G \rho_{e}}{3}} \text { Or } \omega_{e}^{2}=\frac{8 \pi G \rho_{e}}{3} \\
\text { Density, } \rho_{\mathrm{e}}=\frac{3 \omega_{\mathrm{e}}^{2}}{8 \pi \mathrm{G}}
\end{gathered}
$$

In real time, this obtained density may or may not be equal to the actual density. But the ratio $\frac{8 \pi G \rho_{\text {real }}}{3 \omega_{\text {real }}^{2}}$ may have some physical significance. The most important point to be noted here, is that, as far as dimensions and units are considered, from equation (4), it is very clear that, proportionality constant being $\frac{3}{8 \pi G}$,

$$
\text { density } \propto(\text { angular velocity })^{2}
$$

Equation (4) is similar to "flat model concept" of cosmic "critical density"

$$
\rho_{c}=\frac{3 H_{t}^{2}}{8 \pi G}
$$

Comparing equations (4) and (6) dimensionally and conceptually, i.e. 


$$
\begin{gathered}
\rho_{e}=\frac{3 \omega_{e}^{2}}{8 \pi G} \text { with } \rho_{\mathrm{c}}=\frac{3 H_{\mathrm{t}}^{2}}{8 \pi \mathrm{G}} \\
H_{t}^{2} \rightarrow \omega_{\mathrm{e}}^{2} \text { and } H_{t} \rightarrow \omega_{e}
\end{gathered}
$$

It is very clear that, dimensions of 'Hubble's constant' must be 'radian/second'. In any physical system under study, for any one 'simple physical parameter' there will not be two different units and there will not be two different physical meanings. This is a simple clue and brings "cosmic rotation" into picture. This is possible in a closed universe only. Cosmic models that depend on this "critical density" may consider 'angular velocity of the universe' in the place of 'Hubble's constant'. In the sense, with a great confidence 'cosmic rotation' can be included in the existing models of cosmology. Then the term 'critical density' appears to be the 'volume density' of the closed and expanding universe.

\section{To Re-Interpret the Hubble's Law}

It may be noted that, increased redshifts and increased distances forced Edwin Hubble to propose the Hubble's law Since galaxy is not a point particle and if light is coming from the atoms of the gigantic galaxy, then cosmic redshift can be interpreted as an index of the galactic atomic 'light emission mechanism'. In no way it seems to be connected with 'galaxy receding'. If it is possible to show that, (from the observer) observed older galaxy's distance increases with its 'age', then the concepts 'galaxy receding' and 'accelerating universe' can be put for a revision at fundamental level. Whatever may be the expression, definitions of cosmic red shift seem to be ad-hoc and not basic or original definition of present/current redshift $\left(z_{0}\right)$ seems to be:

$$
z_{0} \cong \frac{E_{0}-E_{G}}{E_{0}} \cong \frac{\lambda_{G}-\lambda_{0}}{\lambda_{G}} \cong\left(z_{x}\right)_{0} \leq 1 \text {. (say) }
$$

But not

$$
z_{0} \cong \frac{E_{0}-E_{G}}{E_{G}} \cong \frac{\lambda_{G}-\lambda_{0}}{\lambda_{0}} \cong\left(z_{y}\right)_{0} \text { (say) }
$$

With reference to the current definition of $z \cong\left(z_{y}\right)_{0}$, proposed $z \cong\left(z_{x}\right)_{0}$ can be expressed as follows.

$$
\left(z_{x}\right)_{0} \cong \frac{\left(z_{y}\right)_{0}}{1+\left(z_{y}\right)_{0}}
$$

Here $E_{0} \cong \frac{h c}{\lambda_{0}}$ is the energy of photon at our galaxy/laboratory and $E_{G} \cong \frac{h c}{\lambda_{G}}$ is the energy of photon at the observed galaxy when it was emitted. Similarly $\lambda_{G}$ is the wave length of light received from observed galaxy and $\lambda_{0}$ is the wave length of light in laboratory. Even though both relations are ad-hoc and not absolute definitions, compared to relation (10), relation (9) seems to be somewhat reliable. Very interesting thing is that, when redshift is very small (up to $z \approx 0.01$ ), both relations almost all will give the same result. Important point to be noticed is that, by Hubble's time the maximum redshift noticed was 0.003 and was less than 0.01 . One should not ignore this fact. Now the fundamental question to be answered is: which relation is correct: either relation (9) or relation (10)? Note that, present red shift $\left(z_{0}\right)$ will be directly proportional to age difference between our galaxy and observed galaxy or time taken by light to reach our galaxy from the observed $\operatorname{galaxy}(\Delta t)$. Thus $z_{0} \propto \Delta t$ and

$$
z_{0} \cong H_{0} \Delta t .
$$

Here $H_{0}$ is the proportionality constant. In this way $H_{0}$ can be incorporated directly. Time taken by light to reach our galaxy or the age difference of our galaxy and observed galaxy can be expressed as,

$$
\begin{gathered}
\Delta t \cong \frac{z_{0}}{H_{0}} . \\
c \Delta t \cong z_{0} \cdot \frac{c}{H_{0}} .
\end{gathered}
$$

To confirm this, absolute methods (that are free from redshift) for estimating galaxy age can be considered. Then the basic and original definition of 'galaxy receding' and 'accelerating universe' concepts can be eliminated and a 'decelerating or expanded universe' concept can be continued without any difficulty. Hence with redshift concept - one may not be able to understand the actual rate of cosmic expansion and actual cosmic geometry $[45,46]$.

\section{Possible Assumptions}

The possible assumptions in unified cosmic physics can be expressed in the following way.

A) With reference to the elementary charge and with mass similar to the Planck mass, a new mass unit can be constructed in the following way. It can be called as the Coulomb mass.

$$
\begin{aligned}
\left(M_{C}\right)^{ \pm} & \cong \sqrt{\frac{e^{2}}{4 \pi \varepsilon_{0} G}} \cong 1.859210775 \times 10^{-9} \mathrm{Kg} \\
& \cong 1.042941 \times 10^{18} \mathrm{GeV} / \mathrm{c}^{2}
\end{aligned}
$$


It represents the characteristic mass of elementary charge in unification program. It can be considered as the seed of galactic matter or galactic central black hole. It can also be considered as the seed of any cosmic structure.

B) At any time Hubble length $\left(c / H_{t}\right)$ can be considered as the gravitational or electromagnetic interaction range.

C) At any time, $H_{t}$ being the angular velocity, universe can be considered as a growing and light speed rotating primordial black hole. Thus at any given cosmic time,

$$
R_{t} \cong \frac{2 G M_{t}}{c^{2}} \cong \frac{c}{H_{t}} \text { and } M_{t} \cong \frac{c^{3}}{2 G H_{t}}
$$

when $M_{t} \rightarrow M_{C}, R_{C} \cong \frac{2 G M_{C}}{c^{2}}$ and $H_{C} \cong \frac{c}{R_{C}} \cong \frac{c^{3}}{2 G M_{C}}$ can be considered as the characteristic initial physical measurements of the universe. Here the subscript $C$ refers to the initial conditions of the universe and can be called as the Coulomb scale. Similarly $R_{0} \cong \frac{2 G M_{0}}{c^{2}} \cong \frac{c}{H_{0}}$ and $\mathrm{M}_{0} \cong \frac{c^{3}}{2 G H_{0}}$ can be considered as the characteristic current physical measurements of the universe.

D) Reduced Planck's constant or the characteristic angular momentum of the revolving electron increases with cosmic time whereas the Planck's constant can be considered as a cosmological constant [47].

E) Characteristic nuclear size [48-52] increases with cosmic time. In this regard, to a great surprise, if $\mathrm{H}_{0}$ is close to $71 \mathrm{~km} / \mathrm{sec} / \mathrm{Mpc}$, it is noticed that,

$$
\frac{G \sqrt{M_{0} \sqrt{m_{p} m_{e}}}}{c^{2}} \cong 1.37 \times 10^{-15} \mathrm{~m}
$$

F) Atomic gravitational constant $G_{A}$ is squared Avogadro number times the classical gravitational constant $G$.

$$
\frac{G_{A}}{G} \cong N_{A}^{2} \text { and } G_{A} \cong N_{A}^{2} G
$$

where $N_{A}$ is the Avogadro number [2,52].

G) Similar to the classical force limit $\left(\frac{c^{4}}{G}\right)$, with $G_{A} \cong N_{A}^{2} G$, a binding force of the following form can be introduced into atomic system.

$$
F_{X} \cong \frac{c^{4}}{G_{A}}
$$

Note that if $Z$ is the number of protons in the nucleus then $Z\left(\frac{c^{4}}{G_{A}}\right)$ can be considered as the force acting on any electron. It plays a very interesting role in modified Super symmetric Higgs fermion \& boson, fitting of electron, muon, tau, proton and neutron rest masses and nuclear binding energy coefficients [2].

\section{Cosmic Thermal and Physical Parameters and Relation between the Fundamental Thermal Radiation Constants}

At any given cosmic time, thermal energy density can be expressed as follows.

$$
a T_{t}^{4} \cong\left[1+\ln \left(\frac{M_{t}}{M_{C}}\right)\right]^{-2}\left(\frac{3 H_{t}^{2} c^{2}}{8 \pi G}\right)
$$

Matter-energy density can be considered as the geometric mean density of volume energy density and the thermal energy density and it can be expressed as follows.

$$
\begin{aligned}
& \left(\rho_{m}\right)_{t} c^{2} \cong \sqrt{\left(\frac{3 H_{t}^{2} c^{2}}{8 \pi G}\right)\left(a T_{t}^{4}\right)} \\
& \cong\left[1+\ln \left(\frac{M_{t}}{M_{C}}\right)\right]^{-1}\left(\frac{3 H_{t}^{2} c^{2}}{8 \pi G}\right)
\end{aligned}
$$

Thus at present [53-56],

$$
\begin{aligned}
a T_{0}^{4} \cong\left[1+\ln \left(\frac{M_{0}}{M_{C}}\right)\right]^{-2}\left(\frac{3 H_{0}^{2} c^{2}}{8 \pi G}\right) \\
\left(\rho_{m}\right)_{0} c^{2} \cong \sqrt{\left(\frac{3 H_{0}^{2} c^{2}}{8 \pi G}\right)\left(a T_{0}^{4}\right)} \\
\cong\left[1+\ln \left(\frac{M_{0}}{M_{C}}\right)\right]^{-1}\left(\frac{3 H_{0}^{2} c^{2}}{8 \pi G}\right)
\end{aligned}
$$

It is also possible to express the CMBR wavelength in the following way [2].

$$
\begin{aligned}
& \left(\lambda_{m}\right)_{0} \cong \sqrt{\left[1+\ln \left(\frac{M_{0}}{M_{C}}\right)\right] \cdot\left(\frac{M_{0}}{M_{C}}\right) \cdot\left(\frac{b k_{B} G}{2 c^{4}}\right)} \\
& \cong 1.064 \mathrm{~mm}
\end{aligned}
$$

In this approach it is noticed that,

$$
\frac{8 \pi^{5}}{15}\left(\frac{k_{B}^{3} b^{3}}{h^{3} c^{3}}\right) \cong 1.3333995 \cong \frac{4}{3} .
$$

Thus radiation energy density constant $a$ can be expressed as follows. 


$$
a \cong \frac{4}{3} \cdot \frac{k_{B}}{b^{3}}
$$

Based on this idea and considering the beginning case of cosmic evolution, i.e. $a T_{C}^{4} \cong \frac{3 H_{C}^{2} c^{2}}{8 \pi G}$, with $3 \%$ error, Wien's displacement constant can be expressed as follows.

$$
b \cong \frac{512 \pi}{9} \frac{e^{2}}{4 \pi \varepsilon_{0} k_{B}} \cong 2.986457 \times 10^{-3}{ }^{0} \mathrm{~K} \cdot \mathrm{m}
$$

Combining these results with Planck's quantum theory, it is possible to express the Planck's constant as follows.

$$
h \cong\left(\frac{2 \pi^{5}}{5}\right)^{\frac{1}{3}} \frac{b k_{B}}{c} \cong\left(\frac{2 \pi^{5}}{5}\right)^{\frac{1}{3}} \cdot \frac{512 \pi}{9} \cdot \frac{e^{2}}{4 \pi \varepsilon_{0} c}
$$

Please note that $\left(\frac{2 \pi^{5}}{5}\right)^{\frac{1}{3}} \cong 4.9652$.

\section{The Cosmological Fine Structure Ratio}

In atomic and nuclear physics, the fine-structure ratio $(\alpha)$ is a fundamental physical constant namely the coupling constant characterizing the strength [57,58] of the electromagnetic interaction. Being a dimensionless quantity, it has a constant numerical value in all systems of units. If $\rho_{0} c^{2}$ is the present cosmic critical energy density and $a T_{0}^{4}$ is the present cosmic thermal energy density, it is noticed that,

$$
\ln \sqrt{\frac{a T_{0}^{4}}{\rho_{0} c^{2}} \cdot \frac{4 \pi \varepsilon_{0} G M_{0}^{2}}{e^{2}}} \cong\left(\frac{1}{\alpha}\right)
$$

At present, if $H_{0}$ is close to $71 \mathrm{~km} / \mathrm{sec} / \mathrm{Mpc}$ and $T_{0} \cong 2.725{ }^{0} \mathrm{~K}$, obtained value of $(1 / \alpha)_{0}$ is 137.04773 . Note that, from unification point of view, till today role of dark energy or dark matter is unclear and undecided. Their laboratory or physical existence is also not yet confirmed. In this critical situation this application can be considered as a key tool in particle cosmology. Note that large dimensionless constants and compound physical constants reflect an intrinsic property of nature. Above relation takes the following form.

$$
\ln \sqrt{\frac{2 \pi}{3} \cdot \frac{4 \pi \varepsilon_{0}\left(a T_{0}^{4}\right) c^{4}}{e^{2} H_{0}^{4}}} \cong\left(\frac{1}{\alpha}\right)_{0}
$$

After simplification, it can be interpreted as follows. Total thermal energy in the present Hubble volume can be expressed as,

$$
\begin{aligned}
& \left(E_{T}\right)_{0} \cong a T_{0}^{4} \cdot \frac{4 \pi}{3}\left(\frac{c}{H_{0}}\right)^{3} \\
& \cong\left[\left(1+\ln \left(\frac{M_{0}}{M_{C}}\right)\right)^{-2} \frac{3 H_{0}^{2} c^{2}}{8 \pi G}\right] \cdot \frac{4 \pi}{3}\left(\frac{c}{H_{0}}\right)^{3}
\end{aligned}
$$

If $\left(c / H_{0}\right)$ is the present electromagnetic interaction range, then present electromagnetic potential can be expressed as

$$
\left(E_{e}\right)_{0} \cong \frac{e^{2}}{4 \pi \varepsilon_{0}\left(c / H_{0}\right)}
$$

Now inverse of the present fine structure ratio can be expressed as

$$
\left(\frac{1}{\alpha}\right)_{0} \cong \ln \sqrt{\frac{\left(E_{T}\right)_{0}}{2\left(E_{e}\right)_{0}}}
$$

Here, in the RHS, denominator ' 2 ' may be a representation of total thermal energy in half of the cosmic sphere or thermal energy of any one pole of the cosmic sphere. This is a simple and direct application of the proposed assumptions. Thus at any cosmic time,

$$
\left(\frac{1}{\alpha}\right)_{t} \cong \ln \sqrt{\frac{\left(E_{T}\right)_{t}}{2\left(E_{e}\right)_{t}}}
$$

By any reason, at the initial conditions if thermal energy density equals to $\frac{3 H_{C}^{2} c^{2}}{8 \pi G},\left(\frac{1}{\alpha}\right)_{C} \rightarrow 0$. This is a very surprising and interesting result and needs a critical analysis.

\section{The Cosmic Red Shift, Discrete Atomic Force and Important Cosmological Results in Atomic, Nuclear and Quantum Physics}

\subsection{About the Current Cosmic Red Shift}

It may be noted that, increased redshifts and increased distances forced Edwin Hubble to propose the Hubble's law. Since galaxy is not a point particle and if light is coming from the atoms of the gigantic galaxy, then cosmic redshift can be interpreted as an index of the galactic atomic 'light emission mechanism'. If it is possible to show that, observed older galaxy's distance increases with its 'age', then the concepts 'galaxy receding' and 'accelerating universe' can be put for a revision at fundamental level.' Hubble's law can be reinterpreted in the following way.

1. It can be suggested that, as cosmic time increases $\hbar_{t}$ magnitude increases. $d \hbar / d t$ or $d(1 / \alpha) / d t$ can be considered as a measure of cosmic rate of expansion. 
2. During cosmic evolution, as cosmic time increases, hydrogen atom emits photons with increased quanta of energy. Thus past light quanta emitted from old galaxy will have less energy and show a red shift with reference to our galaxy.

3. During journey light quanta will not lose energy and there will be no change in light wavelength.

4. At any given cosmic time, for any galaxy current cosmic redshift can be expressed in the following way.

$$
\begin{aligned}
& z_{0} \cong \frac{\left(E_{\text {photon }}\right)_{0}-\left(E_{\text {photon }}\right)_{t}}{\left(E_{\text {photon }}\right)_{0}} \cong 1-\left(\frac{\lambda_{0}}{\lambda_{G}}\right) \\
& \cong 1-\left(\frac{\hbar_{t}}{\hbar_{0}}\right)^{2} \cong 1-\left(\frac{H_{0}}{H_{t}}\right) \leq 1 \\
& 1-\left(\frac{\hbar_{t}}{\hbar_{0}}\right)^{2} \cong 1-\left(\frac{H_{0}}{H_{t}}\right) \\
& \rightarrow \hbar_{t} \cong \sqrt{\frac{\lambda_{0}}{\lambda_{G}}} \cdot \hbar_{0} \text { and } H_{t} \cong\left(\frac{\lambda_{G}}{\lambda_{0}}\right) \cdot H_{0}
\end{aligned}
$$

\subsection{About the Current Nuclear Charge Radius}

Please note that so far no nuclear model could explain the origin of strong nuclear force and strong interaction range. From unification point of view it is well established that strong force is $10^{40}$ times stronger than the gravitational force. It is noticed that,

$$
\left(R_{c}\right)_{0} \cong\left(\frac{\hbar c}{G_{A} m_{e}^{2}}\right)^{2}\left(\frac{2 G_{A} m_{e}}{c^{2}}\right) \cong 1.21565 \mathrm{fm}
$$

$\left(R_{c}\right)_{0}$ can be considered as the present nuclear charge radius. It's a discovery and can be considered as a definition also. It can also be expressed in the following way.

$$
\begin{aligned}
& \left(R_{c}\right)_{0} \cong \frac{1}{N_{A}^{2}}\left(\frac{m_{p}}{m_{e}}\right)^{2}\left(\frac{c}{H_{0}}\right) \cong \frac{1}{N_{A}^{2}}\left(\frac{m_{p}}{m_{e}}\right)^{2}\left(\frac{2 G M_{0}}{c^{2}}\right) \\
& \cong\left(\frac{G m_{p}^{2}}{G_{A} m_{e}^{2}}\right)\left(\frac{2 G M_{0}}{c^{2}}\right) \geqq .21 \text { to } 1.22 \mathrm{fm}
\end{aligned}
$$

If one is willing to consider $\hbar$ as a cosmological increasing variable then the above two relations can be coupled to give a single meaning that nuclear charge radius is a cosmological increasing variable.

\subsection{To Fit the Semi Empirical Mass Formula (SEMF) Binding Energy Coefficients in a Cosmological Approach}

The semi-empirical mass formula (SEMF) is used to approximate the mass and various other properties of an atomic nucleus $[59,60]$. As the name suggests, it is based partly on theory and partly on empirical measurements. Based on the 'least squares fit', volume energy coefficient is $a_{v}=15.78 \mathrm{MeV}$, surface energy coefficient is $a_{s}=18.34$ $\mathrm{MeV}$, coulombic energy coefficient is $a_{c}=0.71 \mathrm{MeV}$, asymmetric energy coefficient is $a_{a}=23.21 \mathrm{MeV}$ and pairing energy coefficient is $a_{p}=12 \mathrm{MeV}$. The semi empirical mass formula is

$$
B E \cong A a_{v}-A^{\frac{2}{3}} a_{s}-\frac{Z(Z-1)}{A^{\frac{1}{3}}} a_{c}-\frac{(A-2 Z)^{2}}{A} a_{a} \pm \frac{1}{\sqrt{A}} a_{p}
$$

In the semi empirical mass formula energy coefficients can be fitted in the following way. In a unified approach it is noticed that, the energy coefficients are having strong inter-relation with the above cosmological ratio $k_{0} \cong\left(\frac{G_{A} m_{e}^{2}}{\hbar_{0} c}\right) \cong 635.3132$. The interesting semi empirical observations can be expressed in the following way. Advantage of this proposal is that, magnitude of all the binding energy coefficients decreases with the increasing magnitude of $\left(\frac{G_{A} m_{e}^{2}}{\hbar_{t} c}\right)$ and binding energy reaches to zero indicating there was no binding of nucleons in the past. At present it can be suggested that,

$$
\begin{aligned}
& \left(a_{v}\right)_{0}+\left(a_{s}\right)_{0} \cong\left(a_{a}\right)_{0}+\left(a_{p}\right)_{0} \cong \frac{3}{2}\left(a_{a}\right)_{0} \\
& \cong \frac{m_{p} c^{2}}{1+\sqrt{k_{0}}} \cong 35.8045 \mathrm{MeV}
\end{aligned}
$$

Current asymmetric energy coefficient be

$$
\left(a_{a}\right)_{0} \cong \frac{2}{3} \cdot\left(\frac{m_{p} c^{2}}{1+\sqrt{k_{0}}}\right) \cong 23.870 \mathrm{MeV}
$$

Current pairing energy coefficient be

$$
\left(a_{p}\right)_{0} \cong \frac{\left(a_{a}\right)_{0}}{2} \cong \frac{1}{3} \cdot\left(\frac{m_{p} c^{2}}{1+\sqrt{k_{0}}}\right) \cong 11.935 \mathrm{MeV}
$$

Current maximum nuclear binding energy per nucleon be

$$
\left(B_{m}\right)_{0} \cong \frac{1}{4} \cdot\left(\frac{m_{p} c^{2}}{1+\sqrt{k_{0}}}\right) \cong 8.9511 \mathrm{MeV}
$$

Current coulombic energy coefficient be

$$
\left(a_{c}\right)_{0} \cong \sqrt{\alpha_{0}} \cdot\left(B_{m}\right)_{0} \cong 0.7647 \mathrm{MeV}
$$

Current surface energy coefficient be 


$$
\left(a_{s}\right)_{0} \cong 2\left(B_{m}\right)_{0}\left(1+\sqrt{\frac{\left(a_{c}\right)_{0}}{\left(a_{a}\right)_{0}}}\right) \cong 19.504 \mathrm{MeV}
$$

Current volume energy coefficient be

$$
\left(a_{v}\right)_{0} \cong 2\left(B_{m}\right)_{0}\left(1-\sqrt{\frac{\left(a_{c}\right)_{0}}{\left(a_{a}\right)_{0}}}\right) \cong 16.30 \mathrm{MeV}
$$

In table-1 within the range of $(Z=26 ; A=56)$ to $(Z=92 ; A=238)$ nuclear binding energy is calculated and compared with the measured binding energy. Column-3 represents the calculated binding energy and column-4 represents the measured binding energy.

Table 1. SEMF Binding Energy with the Proposed Energy Coefficients

\begin{tabular}{cccc}
\hline$Z$ & $A$ & $(B E)_{\text {cal }}$ in $\mathbf{M e V}$ & $(B E)_{\text {meas }}$ in $\mathbf{M e V}$ \\
\hline 6 & 56 & 492.17 & 492.254 \\
28 & 62 & 546.66 & 545.259 \\
34 & 84 & 727.75 & 727.341 \\
50 & 118 & 1007.76 & 1004.950 \\
60 & 142 & 1184.50 & 1185.145 \\
79 & 197 & 1556.66 & 1559.40 \\
82 & 208 & 1627.11 & 1636.44 \\
92 & 238 & 1805.60 & 1801.693 \\
\hline
\end{tabular}

Proton-nucleon stability relation can be expressed as follows.

$$
\left(\frac{A_{s}}{2 Z}\right)_{0} \cong 1+2 Z\left[\frac{\left(a_{c}\right)_{0}}{\left(a_{s}\right)_{0}}\right]^{2}
$$

where $\left(A_{s}\right)_{0}$ is the stable mass number of $Z$. This is a direct relation. Assuming the proton number $Z$, in general, for all atoms, lower stability can be fitted directly with the following relation. Stable super heavy elements can also be predicted with this relation.

$$
\begin{aligned}
& \quad\left(A_{s}\right)_{0} \cong 2 Z\left[1+2 Z\left[\frac{\left(a_{c}\right)_{0}}{\left(a_{s}\right)_{0}}\right]^{2}\right] \cong 2 Z+Z^{2} * 0.00615 \\
& \text { if } Z=21, A_{s} \cong 44.71 ; \text { if } Z=29, A_{s} \cong 63.17 ; \\
& \text { if } Z=47, A_{s} \cong 107.58 \text {; if } Z=53, A_{s} \cong 123.27 \\
& \text { if } Z=60, A_{s} \cong 142.13 \text {; if } Z=79, A_{s} \cong 196.37 ; \\
& \text { if } Z=83, A_{s} \cong 208.36 \text {; if } Z=92, A_{s} \cong 236.04 ;
\end{aligned}
$$

At the beginning of cosmic evolution, binding energy coefficients and hence binding energy reaches to zero, and the expression $2 Z\left[\frac{\left(a_{c}\right)_{0}}{\left(a_{s}\right)_{0}}\right]^{2} \cong 0$, also reaches to zero, more or the less the proton - nucleon stability takes the following form.

$$
\left(\frac{A_{s}}{2 Z}\right)_{\text {beginning }} \cong 1
$$

As nuclear binding energy was zero at the beginning of cosmic evolution, it can be suggested that, from the beginning of formation of nucleons, in any galaxy, maximum scope is being possible only for the survival of light atoms and this may be the reason for the accumulation and abundance of light atoms in large proportion.

\subsection{To Understand the Absoluteness of the Avogadro Number and Cosmological 'Gram Mole'}

Independent of any system of units, the characteristic relation that connects the 'gram mole' and the unified atomic mass unit can be expressed as follows.

$$
\begin{aligned}
& G_{A}\left(m_{u}^{2}\right)_{0} \cong G\left(M_{x}^{2}\right)_{0} \text { and } \\
& \left(M_{x}\right)_{0} \cong \sqrt{\frac{G_{A}}{G}} \cdot\left(m_{u}\right)_{0} \cong N_{A} \cdot\left(m_{u}\right)_{0}
\end{aligned}
$$

Here $\left(m_{u}\right)_{0}$ is the current unified atomic mass unit and $\left(m_{u} c^{2}\right)_{0} \cong\left(\sqrt{m_{p} m_{n}} c^{2}-\left(B_{\text {Ave }}\right)_{0}\right)+m_{e} c^{2} \cong 931.43 \mathrm{MeV}$ where $\left(B_{\text {Ave }}\right)_{0} \approx 8 \mathrm{MeV}$ is the current average binding energy per nucleon. Current $\left(M_{x}\right)_{0} \approx 0.001 \mathrm{~kg} \approx 1$ gram and may not be exactly 'one' gram'. Accuracy mainly depends upon the current average binding energy per nucleon.

\subsection{About the Discrete Atomic Force}

Note that, in any bound system, 'operating force' only plays a major role in maintaining the 'existence of the bound system' and 'angular momentum' is one of the results. If one is able to make the operating force as discrete, then automatically one can observe a discrete structure like discrete radii, discrete angular momentum and discrete energy levels. If $n=1,2,3, \ldots$ based on the new idea $\left(n N_{A}\right)$, it is possible to introduce a characteristic discrete force magnitude as follows.

$$
\left(F_{X}\right)_{n} \cong \frac{c^{4}}{\left(n N_{A}\right)^{2} G} \cong \frac{c^{4}}{n^{2} G_{A}}
$$

It is having many applications in atomic, nuclear and particle physics. The basic ideas can be stated as follows.

1. Nuclear charge radius increases with cosmic time.

2. Nuclear charge radius is more fundamental than the presently believed 'reduced Planck's constant'.

3. At any given cosmic time, Reduced Planck's constant is a function of increasing nuclear charge radius.

4. Within the hydrogen atom, nuclear charge radius, proton mass and electron mass play a vital role.

5. If nuclear mass and nuclear charge radius both are assumed to play a fundamental role in the formation of 
atom and cause electron to revolve round the nucleus, then reduced Planck's constant can be assumed as cosmological compound atomic physical variable and can be considered as an outcome result of the atomic system but not an input to the atomic system.

6. Proposed discrete force $\left(F_{X}\right)_{n} \cong \frac{c^{4}}{\left(n N_{A}\right)^{2} G} \cong \frac{c^{4}}{n^{2} G_{A}}$ plays a vital role in the observed discrete energy spectrum of Hydrogen atom. It is the root cause of the observed discrete angular momentum of electron.

\subsection{About the Reduced Planck's Constant}

The present quantum of angular momentum of electron can be expressed as follows.

$$
\begin{aligned}
\left(\hbar_{0}\right) & \cong m_{e} c^{2} \sqrt{\left(\frac{G_{A}}{c^{4}}\right) \frac{m_{e}\left(R_{c}\right)_{0}}{2}} \\
& \cong \sqrt{\frac{M_{0}}{m_{e}}}\left(\frac{G m_{p} m_{e}}{c}\right)
\end{aligned}
$$

Here the ratio $\left(\frac{M_{0}}{m_{e}}\right)$ represents the virtual number electrons that may exist in the present Hubble volume of mass $M_{0} \cong c^{3} / 2 G H_{0}$. The present discrete angular momentum of electron can be expressed as follows.

$$
n\left(\hbar_{0}\right) \cong m_{e} c^{2} \sqrt{\left(\frac{n^{2} G_{A}}{c^{4}}\right) \frac{m_{e}\left(R_{c}\right)_{0}}{2}}
$$

Considering the maximum possible number of electrons that may present in any principal quantum shell i.e, $\left(2 n^{2}\right)$, present discrete potential energy of electron in hydrogen atom can be expressed as follows.

$$
\begin{aligned}
& \left(E_{P}\right)_{0} \cong-\left(\frac{\hbar_{0} c}{G_{A} m_{e}^{2}}\right)^{2} \frac{\sqrt{m_{p} m_{e}} \cdot c^{2}}{2 n^{2}} \\
& \cong-\frac{1}{4} \sqrt{\frac{m_{p}}{m_{e}}}\left(\frac{c^{4}}{n^{2} G_{A}}\right)\left(R_{c}\right)_{0}
\end{aligned}
$$

One can find detailed information in our published review article [2] and other published papers [19-27]. Present discrete Bohr radii can be expressed as follows.

$$
\left(a_{n}\right)_{0} \cong 4 \sqrt{\frac{m_{e}}{m_{p}}}\left(\frac{n^{2} G_{A}}{c^{4}}\right) \frac{e^{2}}{4 \pi \varepsilon_{0}\left(R_{c}\right)_{0}}
$$

\subsection{About the rms Radius of Proton}

If $\left(R_{p}\right)_{0}$ is the present rms radius of proton $[51,52]$ to a very good accuracy it is noticed that,

$$
\sqrt{\frac{M_{0}}{m_{e}}} \cong \frac{4 G M_{0}}{c^{2}\left(R_{p}\right)_{0}}
$$

The ratio $\left(\frac{4 G M_{0}}{c^{2}\left(R_{p}\right)_{0}}\right)$ resembles the ratio proposed in theory of bending of light. With reference to the cosmological constancy of Planck's constant and uncertainty relation, it is possible to express the present rms radius of proton as follows.

$$
\left(R_{p}\right)_{0} \cong \frac{4 \pi G m_{p} m_{e}}{h H_{0}} \cong \frac{G m_{p} m_{e}}{(h / 4 \pi) H_{0}}
$$

Alternatively, at present

$$
\frac{G m_{p} m_{e}}{\left(R_{p}\right)_{0} H_{0}} \cong \frac{h}{4 \pi}
$$

To maintain the constancy of $(h / 4 \pi)$ throughout the cosmic time, the product $\left[\left(R_{p}\right)_{0} H_{0}\right]$ can be considered as a cosmological constant. Clearly writing at present or at any cosmic time,

$$
\begin{gathered}
\left(R_{p}\right)_{0} H_{0} \cong\left(R_{p}\right)_{t} H_{t} \cong \text { constant } \\
\frac{G m_{p} m_{e}}{\left(R_{p}\right)_{0} H_{0}} \cong \frac{h}{4 \pi} \cong \frac{G m_{p} m_{e}}{\left(R_{p}\right)_{t} H_{t}}
\end{gathered}
$$

\section{Discussion and Conclusions}

The basic idea of unification is - 1) To minimize the number of physical constants and to merge a group of different fundamental constants into one compound physical constant with appropriate unified interpretation and 2) To merge and minimize various branches of physics. In this journey, the first step is to see the numerical coincidences, second step is to interpret the numerical coincidences and the third step is to synchronize the current interpretations and new interpretations. When the new interpretation disagrees with the current interpretation, generally with the help of emerging science and technology, discrepancies can be resolved with future observations, experiments and analysis.

It can be suggested that, there exists one variable physical quantity in the presently believed atomic and nuclear physical constants and "rate of change" in its magnitude can be considered as a "standard measure" of the present "cosmic rate of expansion". Characteristic nuclear radius, rms radius of proton, strength of electromagnetic interaction and the characteristic reduced Planck's constant all seem to be the cosmological variables and observing the rate of change in their magnitude (on the 
cosmological time scale), the cosmic acceleration can be verified and thus independent of the cosmic red shift and CMBR observations the cosmic geometry can be confirmed from atomic, nuclear and particle physics. Without the advancement of nano-technology or femto-technology this may not be possible. From the above relations it is also clear that, now the black hole universe is expanding in a decelerating mode at a very small rate in such a way that with current technology one cannot measure its deceleration rate.

Finally it can be suggested that there was no big bang and cosmic acceleration and dark energy can be considered as pure mathematical concepts and there exist no physical base behind their confirmation. Now the key leftover things are nucleosynthesis and structure formation. The most important point to be noted here is that, synthesis of elementary physical constants seem to be more important and intrinsic than the 'cosmological nucleosynthesis'. Authors are working on this concept and will be discussed in detail in near future. With the proposed applications it is very clear to say that, without a combined and unified study of cosmology and microscopic physics, one should not deny the concepts of black hole cosmology.

\section{Acknowledgements}

The first author is indebted to professor K. V. Krishna Murthy, Chairman, Institute of Scientific Research on Vedas (I-SERVE), Hyderabad, India and Shri K. V. R. S. Murthy, former scientist IICT (CSIR) Govt. of India, Director, Research and Development, I-SERVE, for their valuable guidance and great support in developing this subject.

\section{References}

[1] Mustapha Ishak, Remarks on the Formulation of the Cosmological Constant/Dark Energy Problems, Found Phys, 37,pp 1470-1498, 2007.

[2] U. V. S. Seshavatharam, S. Lakshminarayana. Microscopic Physical Phenomena in Black Hole Cosmos Rotating at Light Speed. Prespacetime Journal. October 2013, Volume 4, Issue 9, pp. 884-922.

[3] Zhang, Tianxi. Cosmic microwave background radiation of black hole universe. Astrophysics and Space Science, Volume 330,Issue 1, pp 157-165. (2010).

[4] Poplawski, N. J. Radial motion into an Einstein-Rosen bridge. Physics Letters B 687 (23): 110-113. (2010).

[5] Pourhasan R, Afshordi N and Mann R.B. Did a hyper black hole spawn the universe? Nature - International weekly journal of science. 13 September 2013, doi:10.1038/nature.2013.13743, arXiv: $1309.1487 \mathrm{v} 2$.

[6] Hawking S.W. A Brief History of Time. Bantam Dell Publishing Group. 1988

[7] Hawking, S.W.; Ellis, G.F.R. (1973). The Large-Scale
Structure of Space-Time. Cambridge University Press. ISBN 0-521-20016-4.

[8] Hubble E. P, A relation between distance and radial velocity among extra-galactic nebulae, PNAS, 1929, vol. 15, 1929, pp.168-173.

[9] Hubble, E.P, The 200-inch telescope and some problems it may solve. PASP, 59, pp153-167, 1947.

[10] Pathria, R. K. The Universe as a Black Hole. Nature 240 (5379):298-299.doi:10.1038/240298a0 (1972).

[11] Good, I. J. Chinese universes. Physics Today 25 (7): 15. July. doi:10.1063/1.3070923 (1972).

[12] Poplawski, N. J. The universe as a black hole in isotropic coordinates.arXiv:0901.0215v1.

[13] Zhang, Tianxi. A New Cosmological Model: Black Hole Universe. Progress in Physics, 3: 3-11, (2009).

[14] Zhang, Tianxi. Quasar Formation and Energy Emission in Black Hole Universe. Progress in Physics, 3: 48-53, (2012).

[15] Joel Smoller and Blake Temple. Shock-wave cosmology inside a black hole. Proc Natl Acad Sci U S A. September 30 100(20): 1121611218. (2003).

[16] Andy Gardner, Joseph P. Conlon. Cosmological natural selection and the purpose of the universe. Complexity Volume 18, Issue 5, pages 48-56, May/June 2013

[17] Chul-Moon Yoo et al. Black Hole Universe. Time evolution. Phys. Rev. Lett. 111, 161102(2013).

[18] Poplawski, N. J. Energy and momentum of the Universe.arXiv:1305.6977v1.

[19] U. V.S. Seshavatharam and S. Lakshminarayana. Black hole Cosmos and the Micro Cosmos. International Journal of Advanced Astronomy, 1 (2) (2013) 37-59.

[20] U. V. S. Seshavatharam. Physics of rotating and expanding black hole universe. Progress in Physics. April, p 7-14, (2010).

[21] U.V.S. Seshavatharam. The Primordial Cosmic Black Hole and the Cosmic Axis of Evil. International Journal of Astronomy, 1(2): 20-37, (2012).

[22] U. V. S. Seshavatharam and S. Lakshminarayana. The reduced Planck's constant, Mach's principle, cosmic acceleration and the Black hole universe. Journal of Physical Science and Application 2 (10) (2012) 441-447.

[23] U. V. S. Seshavatharam, S. Lakshminarayana, B.V.S.T. Sai. Unified Concepts in Cosmic, Atomic and Nuclear Physics.Global Journal of Science Frontier Research ( A ) Volume XIII Issue I Version I Year 2012

[24] U. V. S. Seshavatharam, S. Lakshminarayana, Hubble Volume and the Fundamental Interactions, International Journal of Astronomy, Vol. 1 No. 5, 2012, pp. 87-100.

[25] U. V. S. Seshavatharam, S. Lakshminarayana, B.V.S.T. Sai. Is red shift an index of galactic'atomic light emission' mechanism? International Journal of Physics, Vol. 1, No.3, 49-64, (2013).

[26] U. V. S. Seshavatharam, S. Lakshminarayana, B.V.S.T. Sai. Nucleus, Atom and the Universe a combined study. 
International Journal of Advanced Astronomy, 1 (1) , 1-12 (2013).

[27] Seshavatharam U.V. S. and Lakshminarayana. To confirm the existence of Black hole cosmology. International Journal of Advanced Astronomy, 2 (1), 21-36, 2013

[28] Michael J. Longo, Detection of a Dipole in the Handedness of Spiral Galaxies with Redshifts z 0.04, Phys. Lett. B 699, 224-229 2011.

[29] S.-C. Su and M.-C. Chu. Is the universe rotating? Astrophysical Journal, 703 354. 2009.

[30] J. D. McEwen et al. Bayesian analysis of anisotropic cosmologies: Bianchi VIIh and WMAP.Mon. Not. R. Astron. Soc. 000, 1-15 (2013). arXiv:1303.3409v1.

[31] L. M. Chechin. On the Modern Status of the Universe Rotation Problem. Journal of Modern Physics, 2013, 4, 126132.

[32] C Sivaram and Kenath Arun, Primordial Rotation of the Universe, Hydrodynamics, Vortices and Angular Momenta of Celestial Objects. The Open Astronomy Journal, 2012, 5, $7-11$

[33] Sidharth, B.G. Is the Universe Rotating? Prespacetime Journal. October 2010, Vol. 1, Issue 7, pp. 1168-1173.

[34] Marcelo Samuel Berman, Fernando de Mello Gomide. Local and Global Stability of the Universe. Journal of Modern Physics, 2013, 4, 7-9

[35] Robert V Gentry. New Cosmic Center Universe Model Matches Eight of Big Bang's Major Predictions Without The F-L Paradigm. CERN preprint, EXT-2003-022, 14 Apr 2003.

[36] G. Chapline et al. Tommy Gold Revisited: Why Does Not The Universe Rotate? AIP Conf.Proc.822:160-165, 2006. http://arxiv.org/abs/astro-ph/0509230.

[37] Dmitri Rabounski. On the Speed of Rotation of Isotropic Space: Insight into the Redshift Problem. The Abraham Zelmanov Journal, Vol. 2, 2009, 208-223.

[38] Kurt Godel. Rotating Universes in General Relativity Theory. Proceedings of the international Congress of Mathematicians in Cambridge, 1: 175-81, 1950.

[39] S.W. Hawking. On the rotation of the universe. Mon. Not. Royal. Astr. Soc. 142, 129-141.1969.

[40] M. Novello and M. J. Reboucas. Rotating universe with successive causal and noncausal regions. Phys. Rev. D 19, 2850-2852 (1979)

[41] Barrow J D, Juszkiewicz R, Sonoda DH. Universal rotation - How large can it be? Mon Not R AstronSoc 1985; 213: 917.

[42] M. Surdin. The Rotating Universe. Physics Essays: September 1995, Vol. 8, No. 3, pp. 282-284.

[43] Christopher S. Reynolds. Astrophysics: Black holes in a spin Nature. 494, 432-433 (28 February 2013)
[44] U. V. S. Seshavatharam. Light speed rotating black holes: The special holes. International Journal of Advanced Astronomy. 1 (1), 13-20 (2013).

[45] Marmet. A New Non-Doppler Redshift. Physics Essays, 1(1):24-32, 1988. Online

[46] Louis Marmet. On the Interpretation of Red-Shifts: A Quantitative Comparison of Red-Shift Mechanisms. www.marmet.org/louis/index.html

[47] U. V. S. Seshavatharam and S. Lakshminarayana. Is Planck's constant a cosmological variable? International Journal of Astron-omy, 2(1): 11-15 DOI: 10.5923/j.astronomy. 20130201.02 (2013).

[48] Recami E. Elementary Particles as Micro-Universes, and "Strong Black-holes": A Bi-Scale Approach to Gravitational and Strong Interactions. Preprint NSF-ITP-02-94. posted in the arXives as the e-print physics/0505149, and references therein.

[49] Geiger $H$ and Marsden E. On a diffuse reaction of the particles. Proc. Roy. Soc., Ser. A 82: 495-500, 1909.

[50] H. Yukawa. On the Interaction of Elementary Particles. Proc. Phys. Math. Soc. Jap. 17 (48). 1935

[51] Michael O. Distler et al. The RMS Charge Radius of the Proton and Zemach Moments. Phys. Lett.B. 696: 343347,2011

[52] P. J. Mohr and B.N. Taylor, CODATA Recommended Values of the Fundamental Physical Constants.2007. http://physics.nist.gov/constants

[53] C. L. Bennett et al, Nine-Year Wilkinson Microwave Anisotropy Probe (WMAP) Observations: Final Maps and Results. Submitted to Astrophysical Journal Supplement Series. http://arxiv.org/abs/1212.5225v1

[54] J. Huchara. Estimates of the Hubble Constant, 2010. Harvard-Smithsonian Center for Astrophysics. http://hubble.plot.dat

[55] W. L. Freedman et al. Final Results from the Hubble Space Telescope Key Project to Measure the Hubble Constant. The Astrophysical Journal 553 (1): 47-72. 2001.

[56] J. Beringer et al. Particle Data Group. Phys. Rev. D86, 010001 (2012)

[57] J.K. Webb et al. Indications of a spatial variation of the fine structure constant. Physical Review letters, 107 (19) 2011

[58] Jon O'Bryan et al. Constraints on Spatial Variations in the Fine-Structure constant from Planck. http://arxiv.org/pdf/1306.1232v1.pdf

[59] Roy Chowdhury et al. Modified Bethe-Weizsacker mass formula with isotonic shift and new driplines. Mod. Phys. Lett. A20 (2005) p.1605-1618

[60] W. D. Myers et al. Table of Nuclear Masses according to the 1994 Thomas-Fermi Model.(from nsdssd.lbl.gov). 